\title{
Novel technique for distributed fibre sensing based on coherent Rayleigh scattering measurements of birefringence
}

\author{
Xin Lu*, Marcelo A. Soto, Luc Thévenaz \\ EPFL Swiss Federal Institute of Technology, Institute of Electrical Engineering, \\ SCI STI LT, Station 11, CH-1015 Lausanne, Switzerland \\ *E-mail: xin.lu@epfl.ch
}

\begin{abstract}
A novel distributed fibre sensing technique is described and experimentally validated, based on birefringence measurements using coherent Rayleigh scattering. It natively provides distributed measurements of temperature and strain with more than an order of magnitude higher sensitivity than Brillouin sensing, and requiring access to a single fibre-end. Unlike the traditional Rayleigh-based coherent optical time-domain reflectometry, this new method provides absolute measurements of the measurand and may lead to a robust discrimination between temperature and strain in combination with another technique. Since birefringence is purposely induced in the fibre by design, large degrees of freedom are offered to optimize and scale the sensitivity to a given quantity. The technique has been validated in 2 radically different types of birefringent fibres - elliptical-core and Panda polarization-maintaining fibres - with a good repeatability.
\end{abstract}

Keywords: Fibre optics, optical fibre sensors, distributed fibre sensing, Rayleigh scattering, birefringence.

\section{INTRODUCTION}

Distributed fibre sensing (DFS) is dedicated to perform a continuous measurement of environmental variables over long distances and has been widely employed in several real field applications. The 3 scattering processes present in optical fibres - Raman, Brillouin and Rayleigh - can be employed to realize DFS, all showing specific advantages and penalties. In a Raman-based DFS, the sole information about temperature is retrieved from the ratio between optical power of spontaneous anti-Stokes and Stokes/Rayleigh waves, which are very weak and therefore limiting the distance range and accuracy. The Brillouin-based systems are less limited from this point of view, but usually require access to both fibre ends and still show limited sensitivity on temperature/strain. Dynamic Brillouin grating (DBG) is an alternative method that provides a sensitivity 50 times higher than a Brillouin sensor by measuring birefringence changes in polarizationmaintaining (PM) fibres ${ }^{1}$, but requires 3 high-power optical waves counter-propagating in the fibre at distinct frequencies.

Coherent optical time domain reflectometry (COTDR) has recently been applied to DFS and demonstrates extremely high sensitivity, equivalent to milli-Kelvin temperature resolution ${ }^{2}$. In such a system, the incident light from a coherent source is randomly reflected by the tiny density variations along the fibre and these backscattered light with random phase will interfere with each other inside the pulse width, leading to a temporal trace with stochastic varying amplitude ${ }^{3}$. The sensing process of the COTDR can be modelled as a weak fibre Bragg grating (FBG) with random amplitude and pitch. Thus the sensitivity of COTDR is intrinsically identical to a FBG, so $\sim 1000$ times larger than a Brillouin sensor. Unfortunately, this technique only provides relative values instead of absolute temperature/strain measurements, while the high sensitivity may be excessive in real situations, requiring a much broader frequency scanning range.

An intermediate sensitivity between Brillouin and Rayleigh systems would be therefore more suitable, which can be obtained from the birefringence induced in optical fibres ${ }^{1}$, moreover providing absolute measurements of temperature/strain. Recently, measurements of the phase birefringence distribution along fibres have been demonstrated using the spectral cross-correlation of coherent Rayleigh traces successively obtained from the 2 orthogonal polarization $a^{2} s^{4}$. In this paper it is proved that this novel technique is able to measure the variation of birefringence induced by environmental changes, based on the same working principle as the DBG. But unlike the dynamic grating, this method requires access to only one fibre-end and moderate optical powers, with possibility to measure low and high birefringence ${ }^{4}$.

The technique is validated for distributed temperature and strain sensing in 2 very different types of highly birefringent fibres, an elliptical-core and a Panda PM fibre. The measurement error induced by the drift in environmental conditions

Sixth European Workshop on Optical Fibre Sensors, edited by Elfed Lewis, Proc. of SPIE Vol. 9916, 991633 - C 2016 SPIE · CCC code: 0277-786X/16/\$18 · doi: 10.1117/12.2236918 
during the acquisition of the 2 traces in the traditional method ${ }^{4}$ becomes a big concern. Here, the Rayleigh traces for the slow and fast axes are acquired in a single shot, so that this environmental perturbation is made totally negligible. High temperature and strain sensitivities are obtained in both PM fibres, but with different relative strengths. Results demonstrate that this technique shows good repeatability over thermal or strain cycles.

\section{THEORY}

In a COTDR system, a highly-coherent optical pulse is launched into a sensing fibre and the Rayleigh light - backscattered by stationary, but randomly placed scattering centres - interferes within the optical pulse generating a random interference pattern that is measured in the time domain. This random interference leads to a speckle-like reflection spectrum (at each fibre position), which is highly dependent on the local refractive index and is measured by scanning the optical frequency of the optical pulses launched into the fibre ${ }^{3}$. Like FBG sensors, this spectral shift results from the refractive index change induced by environmental perturbations. Consequently, the spectral shift of the measured spectrum at a fibre section, and quantified by the cross-correlation of 2 independent measurements, provides the local temperature/strain variation ${ }^{3}$.

In PM fibres, a refractive index difference is intentionally introduced between the two orthogonal axes. It results in similar refractive index profiles with different optical pitch for the 2 polarization eigenaxes, so that the shape of the COTDR traces obtained from the two eigenaxes turns out to be totally dissimilar even at the same optical frequency. Measuring COTDR traces at different frequencies for the two orthogonal polarization axes of the fibre leads to two different local spectra (i.e. at a given fibre location). The spectral correlation of the local COTDR signal can reveal the local birefringence, represented by a shifted correlation peak in the frequency domain ${ }^{4}$. More specifically, coherent Rayleigh traces are obtained within a certain optical frequency range for fast and slow axes, denoted as $R_{f}(z, v)$ and $R_{s}(z, v)$, respectively. At a given position $z_{0}$, the signal fluctuates randomly as a function of frequency. These two local measured spectra are expected to be frequencyshifted with respect to each other, because the fibre birefringence imposes a refractive index difference $\Delta n$ between these two sets of acquisitions and the physical positioning of the scattering centres is identical for the 2 polarizations ${ }^{4}$. Thus, the cross-correlation $\operatorname{Xcorr}\left(z_{0}, \Delta v\right)=R_{s}\left(z_{0}, v\right) * R_{f}\left(z_{0}, v\right)$ will show a spectral peak at a frequency shift $\Delta v=v_{s}-v_{f}$, which is proportional to the fibre phase birefringence $\Delta n=n_{s}-n_{f}$.

$$
n_{s}\left(v_{s}\right) v_{s}=n_{f}\left(v_{f}\right) v_{f} \quad \rightarrow \quad \Delta v=\frac{-v_{f}}{n_{f}^{g}} \Delta n
$$

where $v_{s}, v_{f}$ and $n_{s}, n_{f}$ are the frequencies and refractive indices along the two orthogonal polarization axes (slow and fast axes), and $n_{f}^{g}$ is the group refractive index of the fast axis ${ }^{4}$.

In practice, the optical frequency scanning is realized by employing the sideband generated from a RF modulation; but since the typical birefringence for the PM fibre is around $4 \times 10^{-4}$, a frequency range of $\sim 50 \mathrm{GHz}$ is typically required. Although current microwave source can meet this requirement, this would highly increase the cost of the whole system. An economic and simple way is to perform a precise shift of the source wavelength between the two measurements along the orthogonal polarization axes to roughly meet the phase matching condition expressed in Eq. (1). COTDR traces are then only acquired within a small frequency range so that a comparatively low RF source is enough to secure high precision in the measurements. The actual frequency shift $\Delta v$ is the sum of the optical frequency difference used for the two orthogonally-polarized measurements and the spectral shift obtained from the cross-correlation of the two measurements ${ }^{4}$.

\section{EXPERIMENT AND RESULTS}

The most-typical implementation, as mentioned above and described in Ref. 4, is not the best implementation for sensing because potential environmental perturbations between the consecutive acquisitions of two sets of traces would introduce measurement errors due to the high environmental sensitivity of the basic COTDR technique. To solve this issue, the simultaneous measurement of the Rayleigh backscattered light from both orthogonal axes is required to avoid phase drifts during the time lapse between the two polarization measurements and thus to realize a reliable sensing system.

An experimental setup for single-shot acquisition of the 2 Rayleigh traces from the 2 orthogonal axes is shown in Fig. 1. Two lasers working around $1534 \mathrm{~nm}$ are employed for the fast and slow axis, respectively, and their operating optical frequencies are independently tuned by the driving current, so that their difference roughly compensates the birefringence effect of the tested fibre. The continuous waves from the two lasers are then simply merged. A small fraction of the light is detected by an optical spectrum analyser (OSA) with $5 \mathrm{MHz}$ resolution to monitor the optical frequency of the lasers, 
while most of the light passes through an intensity modulator driven by a pulsed radio frequency (RF) signal ${ }^{5}$. In this way, the frequency sweeping and the conversion of the sidebands into pulses are realized at the same time. The generated optical pulses show extremely high extinction ratio ${ }^{5}$, which is crucial to reduce the coherent noise in the measurements. Then two FBGs are used to select one sideband from each laser. One of the selected sidebands experiences a delay line that is at least

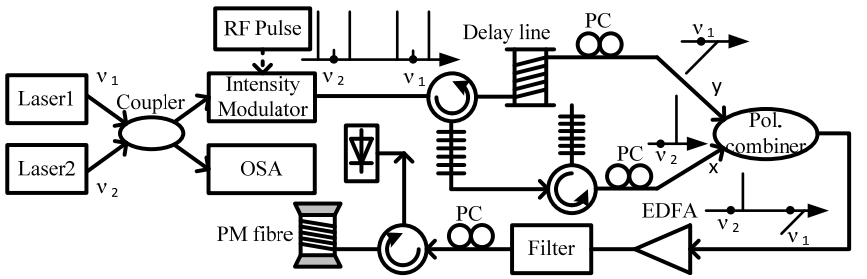

Figure 1. Experimental setup to measure the distributed profile of the phase birefringence of PM fibres. twice the length of the sensing fibre to temporarily offset the optical pulses at different frequencies. Then, using polarization controllers ( $\mathrm{PC}$ ), the polarization of the optical pulses is adjusted, while pulses are recoupled together by a polarization combiner. The peak power of the optical pulses is then boosted by an Erbium-doped fibre amplifier (EDFA) and the amplified spontaneous emission (ASE) noise from the EDFA is suppressed by an optical bandpass filter. A third polarization controller is used to align the orthogonally-polarized pulses to the corresponding axes after passing through the circulator. The Rayleigh scattered light is collected and electrically converted by a $125 \mathrm{MHz}$ photodetector.

The spatial resolution is chosen to be $2 \mathrm{~m}$, which is set by generating optical pulses of $20 \mathrm{~ns}$ width, the shortest limit of the microwave generator. The frequency scanning range is $2 \mathrm{GHz}$ with $5 \mathrm{MHz}$ step. The obtained time-domain traces are averaged 500 times to reduce noise, leading to a global measurement time of $\sim 1 \mathrm{~min}$. To validate the sensing ability of this technique, temperature and strain are independently applied over a few metres at the farthest end of the PM fibre.

A $100 \mathrm{~m}$-long elliptical-core PM fibre and a $20 \mathrm{~m}$-long panda PM fibre have been tested. A $\sim 60 \mathrm{~m}$ delay line is employed for the elliptical-core fibre to offset the optical pulses in the time domain. As a result, the pulse for the fast axis is delayed by $\sim 3.4 \mu \mathrm{s}$, which can be considered to be small enough, thus ensuring that environmental conditions do not significantly change during the acquisition time of the traces along the two orthogonal axes. The two sets of Rayleigh traces from the different axes are cross-correlated ${ }^{4}$ and the local frequency shift is accurately obtained by fitting the correlation peak spectrum with a quadratic curve (see Ref. 6 for details on the impact of a quadratic fitting in a resonance peak). Considering the optical frequency difference of the two lasers, the actual frequency shift corresponding to the birefringence is obtained for all points along the fibre. This frequency shift is therefore supposed to vary in presence of environmental changes.
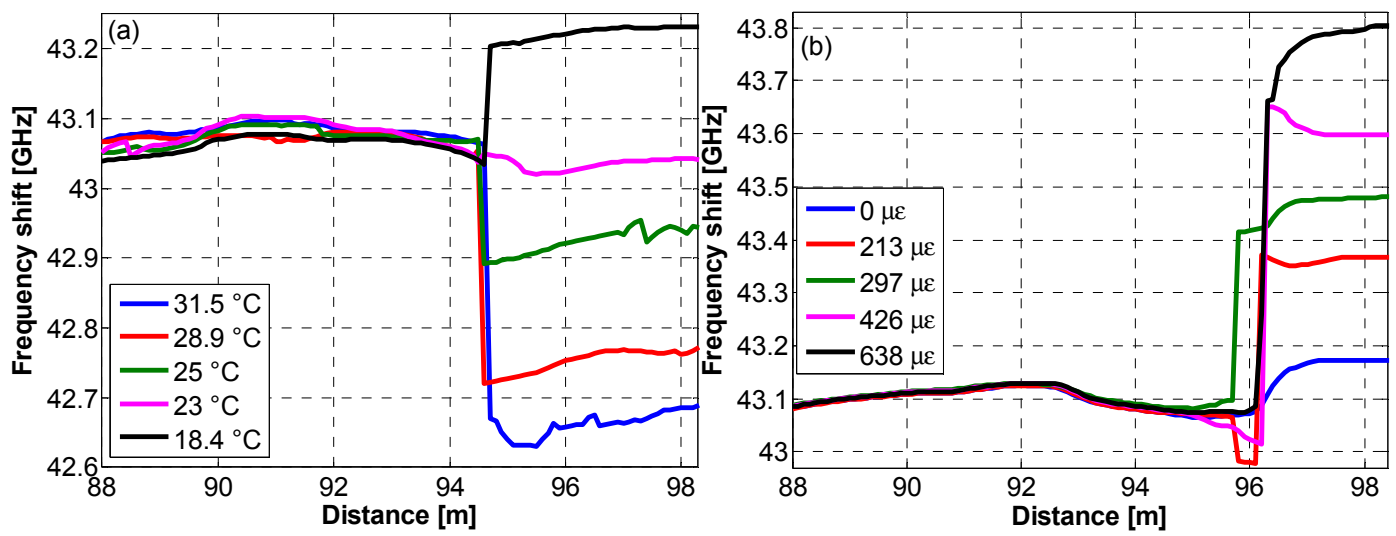

Figure 2. Frequency shift versus distance under different (a) temperature and (b) strain conditions for the elliptical-core PM fibre.

Figure 2 shows the distributed profile of the birefringence-induced frequency shift as a function of distance at the end of the elliptical-core fibre under different temperature (Fig. 2a) and strain (Fig. 2b) conditions. Results clearly demonstrate the ability of the implemented technique to measure birefringence changes induced by temperature and strain variations. In Fig. 2a the frequency shift over the unchanged fibre section (i.e. up to $\sim 94.5 \mathrm{~m}$ ) seems to fluctuate amongst different measurements. This can be explained by the thermal perturbation induced by the thermal bath during the experiment and by the long temporal interval required between measurements to ensure the thermal stabilization of the heated fibre section. On the other hand, the strain measurements requires no overhead time for stabilization, thus minimizing the impact of the environmental thermal fluctuations. Hence the obtained frequency shift fluctuates in the range of $\pm 3 \mathrm{MHz}$ for the nonstrained fibre section, which is lower than the frequency scanning step of $5 \mathrm{MHz}$. 

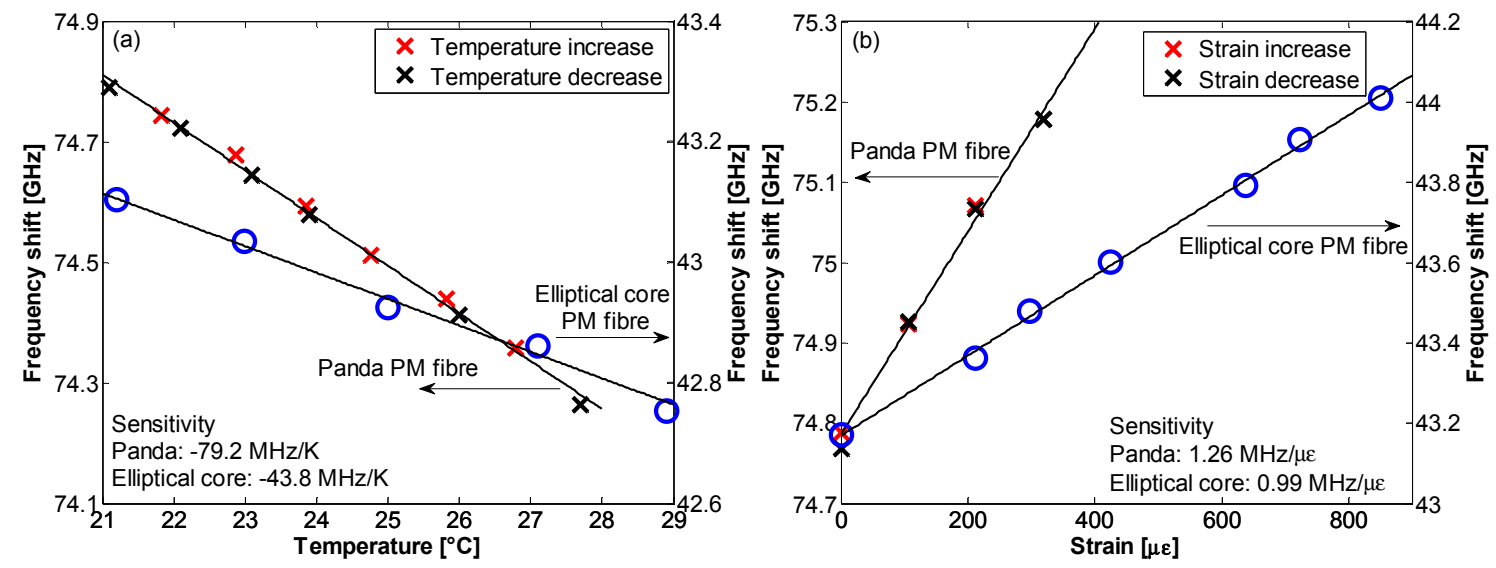

Figure 3. Measured frequency shift versus (a) temperature and (b) strain for the elliptical-core PM fibre and panda PM fibre.

The measured frequency shift changes as a function of the applied temperature and strain are plotted respectively in Fig. 3a and Fig. $3 \mathrm{~b}$ for the elliptical-core PM fibre and Panda fibre. Both figures show the linear dependence of birefringence on temperature and strain, while the slope represents the corresponding sensitivity. All the sensitivities are several tens of times higher than the corresponding sensitivity shown by DFS based on Brillouin scattering. The Panda fibre demonstrates higher sensitivities than the elliptical-core fibre as a result of its higher absolute birefringence. The strain sensitivity roughly scales with the fractional increase in birefringence, while the temperature sensitivity is more enhanced in the Panda fibre than the simple fractional increase, highlighting the influence of the fibre birefringence design for scaling the temperature response. Only a very small discrepancy can be observed while performing a thermal or strain cycle, which remains within the uncertainty range (given by the noise on the measurement ${ }^{6}$ ), demonstrating a high repeatability of this technique.

\section{CONCLUSION}

Birefringence measurements based on COTDR technique are validated for reliable distributed temperature and strain sensing; this constitutes a novel approach for distributed fibre sensing. Experiment results using an elliptical-core and a Panda PM fibre demonstrate that this technique combines the advantages of the birefringence measurements providing absolute temperature and strain measurements, and an order-of-magnitude higher sensitivity than the traditional Brillouin technique. Since the here implemented method is based on coherent Rayleigh scattering, the system is essentially a COTDR sensor, which can also measure environment variations by the spectral cross-correlation of traces from the same axis. Consequently, combining birefringence measurements based on Rayleigh scattering with standard Rayleigh based sensing methods can certainly allow a well-conditioned temperature and strain discrimination. Birefringence can be widely tailored by the structural design of the fibre, opening unprecedented directions in distributed fibre sensing.

The authors thank the support from the European Space Agency (ESA) and the Swiss Space Center (SSC) in the framework of the Call of Order 09 - EPFL-ESA / Contract 4000108465.

\section{REFERENCES}

[1] Kwang, Y. S., Zou, W., He, Z., and Hotate, K., "Optical time-domain measurement of Brillouin dynamic grating spectrum in a polarization-maintaining fiber," Opt. Lett. 34(9), 1381-1383 (2009).

[2] Lu, X., Soto, M. A., and Thevenaz, L., "MilliKelvin resolution in cryogenic temperature distributed fibre sensing based on coherent Rayleigh scattering," Proc. SPIE 9157, 91573R (2014).

[3] Koyamada, Y., et al., "Fiber-optic distributed strain and temperature sensing with very high measurand resolution over long range using coherent OTDR," J. Lightwave Technol. 27(9), 1142-1146 (2009).

[4] Soto, M. A., et al., "Distributed phase birefringence measurements based on polarization correlation in phasesensitive optical time-domain reflectometers," Opt. Express 23(19), 24923-24936 (2015).

[5] Zornoza, A., Olier, D., Sagues, M., and Loayssa, A., "Brillouin distributed sensor using RF shaping of pump pulses," Meas. Sci. Technol., 21(9) 094021 (2010).

[6] Soto, M. A., and Thévenaz, L, "Modeling and evaluating the performance of Brillouin distributed optical fiber sensors," Opt. Express 21(25), 31347-31366 (2013). 